\title{
Impacts of aerosols on the chemistry of atmospheric trace gases: a case study of peroxides and $\mathrm{HO}_{2}$ radicals
}

\author{
H. Liang ${ }^{1}$, Z. M. Chen ${ }^{1}$, D. Huang ${ }^{1}$, Y. Zhao ${ }^{1}$ and Z. Y. Li ${ }^{1,2}$ \\ ${ }^{1}$ State Key Laboratory of Environmental Simulation and Pollution Control, College of \\ Environmental Sciences and Engineering, Peking University, Beijing 100871, China \\ ${ }^{2}$ School of Earth and Space Sciences, Peking University, Beijing 100871, China \\ Correspondence to: Z. M. Chen (zmchen@pku.edu.cn)
}

\section{$\underline{\text { Supplementary material }}$}

Equation S1. Relationship between bulk and surface concentration of $\mathrm{HO}_{2}$

Table S1. Aerosol aqueous phase reactions extended in the box model calculation

Table S2. Sensitivity of $\mathrm{HO}_{\mathrm{x}}$ and $\mathrm{H}_{2} \mathrm{O}_{2}$ concentration to aerosol optical depth (AOD).

Fig.S1. Dependence of j-values on aerosol optical depth (AOD).

Equation S1. Relationship between bulk and surface concentration of $\mathrm{HO}_{2}$

The relationship between bulk and surface concentration of $\mathrm{HO}_{2}$ could be expresses by the following equation (Mao et al., 2013):

$$
\left[\mathrm{HO}_{2}\right]_{\text {surf }}=\frac{P_{\mathrm{HO}_{2}}}{k^{I}}+\left(\left[\mathrm{HO}_{2}\right]_{\text {bulk }}-\frac{P_{\mathrm{HO}_{2}}}{k^{I}}\right) \cdot\left[3\left(\frac{\operatorname{coth} q}{q}-\frac{1}{q^{2}}\right)\right]^{-1}
$$

where $k^{I}$ is the first-order loss constant of $\mathrm{HO}_{2}, P_{\mathrm{HO} 2}$ is the aqueous-phase production rate of $\mathrm{HO}_{2}$, and $q=a\left(k^{I} / D_{\mathrm{aq}}\right)^{1 / 2}$ is the diffuso-reactive parameter, in which a is the radius of the particles, $D_{\mathrm{aq}}$ is the $\mathrm{HO}_{2}$ aqueous phase diffusion constant. According to this correction, the surface concentration is $100 \%$ to $180 \%$ of the bulk concentration, depending on the radius of the particles and the concentrations of TMI. 
Table S1. Aerosol aqueous phase reactions extended in the box model calculation.

\begin{tabular}{|c|c|c|c|}
\hline NO. ${ }^{1}$ & Reactions & $\mathrm{k}_{298}$ & $\mathrm{Ea} / \mathrm{R}$ \\
\hline $\mathrm{R} 1$ & $\mathrm{H}_{2} \mathrm{O}_{2}+\mathrm{Fe}^{2+} \rightarrow \mathrm{Fe}^{3+}+\mathrm{OH}+\mathrm{OH}^{-}$ & $7.0 \times 10^{1}$ & 5050 \\
\hline $\mathrm{R} 2$ & $\mathrm{H}_{2} \mathrm{O}_{2}+\mathrm{FeO}^{2+} \rightarrow \mathrm{Fe}^{3+}+\mathrm{HO}_{2}+\mathrm{OH}^{-}$ & $9.5 \times 10^{3}$ & 2800 \\
\hline R3 & $\mathrm{H}_{2} \mathrm{O}_{2}+\mathrm{Fe}(\mathrm{OH})^{+} \rightarrow \mathrm{Fe}(\mathrm{OH})^{2+}+\mathrm{OH}+\mathrm{OH}^{-}$ & $1.9 \times 10^{6}$ & 6200 \\
\hline R4 & $\mathrm{H}_{2} \mathrm{O}_{2}+\mathrm{Fe}^{3+} \rightarrow \mathrm{Fe}^{2+}+\mathrm{HO}_{2}+\mathrm{H}^{+}$ & $2.0 \times 10^{-3}$ & \\
\hline R5 & $\mathrm{H}_{2} \mathrm{O}_{2}+\mathrm{Fe}(\mathrm{OH})^{2+} \rightarrow \mathrm{Fe}^{2+}+\mathrm{HO}_{2}+\mathrm{H}_{2} \mathrm{O}$ & $2.0 \times 10^{-3}$ & \\
\hline R6 & $\mathrm{O}_{2}^{-}+\mathrm{Fe}^{2+}+2 \mathrm{H}^{+} \rightarrow \mathrm{H}_{2} \mathrm{O}_{2}+\mathrm{Fe}^{3+}$ & $1.0 \times 10^{7}$ & \\
\hline R7 & $\mathrm{O}_{2}^{-}+\mathrm{Fe}^{3+} \rightarrow \mathrm{Fe}^{2+}+\mathrm{O}_{2}$ & $1.5 \times 10^{8}$ & \\
\hline $\mathrm{R} 8$ & $\mathrm{O}_{2}^{-}+\mathrm{Fe}(\mathrm{OH})^{2+} \rightarrow \mathrm{Fe}^{2+}+\mathrm{O}_{2}+\mathrm{OH}^{-}$ & $1.5 \times 10^{8}$ & \\
\hline R9 & $\mathrm{O}_{2}^{-}+\mathrm{Fe}(\mathrm{OH})_{2}^{+} \rightarrow \mathrm{Fe}^{2+}+\mathrm{O}_{2}+2 \mathrm{OH}^{-}$ & $1.5 \times 10^{8}$ & \\
\hline $\mathrm{R} 10$ & $\mathrm{HO}_{2}+\mathrm{Fe}^{2+}+\mathrm{H}^{+} \rightarrow \mathrm{Fe}^{3+}+\mathrm{H}_{2} \mathrm{O}_{2}$ & $1.2 \times 10^{6}$ & 5050 \\
\hline $\mathrm{R} 11$ & $\mathrm{HO}_{2}+\mathrm{FeO}^{2+} \rightarrow \mathrm{Fe}^{3+}+\mathrm{O}_{2}+\mathrm{OH}^{-}$ & $2.0 \times 10^{6}$ & \\
\hline $\mathrm{R} 12$ & $\mathrm{HO}_{2}+\mathrm{Fe}(\mathrm{OH})^{2+} \rightarrow \mathrm{Fe}^{2+}+\mathrm{O}_{2}+\mathrm{H}_{2} \mathrm{O}$ & $1.3 \times 10^{5}$ & \\
\hline $\mathrm{R} 13$ & $\mathrm{OH}+\mathrm{Fe}^{2+} \rightarrow \mathrm{Fe}(\mathrm{OH})^{2+}$ & $4.6 \times 10^{8}$ & 1100 \\
\hline R14 & $\mathrm{OH}+\mathrm{FeO}^{2+}+\mathrm{H}^{+} \rightarrow \mathrm{Fe}^{3+}+\mathrm{H}_{2} \mathrm{O}_{2}$ & $1.0 \times 10^{7}$ & \\
\hline $\mathrm{R} 15$ & $\mathrm{O}_{3}+\mathrm{Fe}^{2+} \rightarrow \mathrm{FeO}^{2+}+\mathrm{O}_{2}$ & $8.2 \times 10^{5}$ & \\
\hline R16 & $\mathrm{FeO}^{2+}+\mathrm{H}_{2} \mathrm{O} \rightarrow \mathrm{Fe}^{3+}+\mathrm{OH}+\mathrm{OH}^{-}$ & $1.3 \times 10^{-2}$ & 4100 \\
\hline R17 & $\mathrm{FeO}^{2+}+\mathrm{Fe}^{2+}+\mathrm{H}_{2} \mathrm{O} \rightarrow 2 \mathrm{Fe}^{3+}+2 \mathrm{OH}^{-}$ & $7.2 \times 10^{4}$ & 842 \\
\hline $\mathrm{R} 18$ & $\mathrm{Cl}_{2}^{-}+\mathrm{Fe}^{2+} \rightarrow \mathrm{Fe}^{3+}+2 \mathrm{Cl}^{-}$ & $1.0 \times 10^{7}$ & 3060 \\
\hline R19 & $\mathrm{O}_{2}^{-}+\mathrm{Fe}\left(\mathrm{SO}_{4}\right)^{+} \rightarrow \mathrm{Fe}^{2+}+\mathrm{SO}_{4}{ }^{2-}+\mathrm{O}_{2}$ & $1.5 \times 10^{8}$ & \\
\hline $\mathrm{R} 20$ & $\mathrm{HO}_{2}+\mathrm{Fe}\left(\mathrm{SO}_{4}\right)^{+} \rightarrow \mathrm{Fe}^{2+}+\mathrm{SO}_{4}^{2-}+\mathrm{O}_{2}+\mathrm{H}^{+}$ & $1.0 \times 10^{3}$ & \\
\hline $\mathrm{R} 21$ & $\mathrm{Fe}^{3+}+\mathrm{SO}_{4}{ }^{2-} \rightarrow \mathrm{Fe}\left(\mathrm{SO}_{4}\right)^{+}$ & $3.2 \times 10^{3}$ & \\
\hline $\mathrm{R} 22$ & $\mathrm{Fe}\left(\mathrm{SO}_{4}\right)^{+} \rightarrow \mathrm{Fe}^{3+}+\mathrm{SO}_{4}^{2-}$ & $2.7 \times 10^{1}$ & \\
\hline $\mathrm{R} 23$ & $\mathrm{OH}+\mathrm{Cu}^{+} \rightarrow \mathrm{Cu}^{2+}+\mathrm{OH}^{-}$ & $3.0 \times 10^{9}$ & \\
\hline $\mathrm{R} 24$ & $\mathrm{O}_{2}+\mathrm{Cu}^{+} \rightarrow \mathrm{Cu}^{2+}+\mathrm{O}_{2}^{-}$ & $4.6 \times 10^{5}$ & \\
\hline $\mathrm{R} 25$ & $\mathrm{H}_{2} \mathrm{O}_{2}+\mathrm{Cu}^{+} \rightarrow \mathrm{Cu}^{2+}+\mathrm{OH}+\mathrm{OH}^{-}$ & $7.0 \times 10^{3}$ & \\
\hline $\mathrm{R} 26$ & $\mathrm{HO}_{2}+\mathrm{Cu}^{+}+\mathrm{H}^{+} \rightarrow \mathrm{Cu}^{2+}+\mathrm{H}_{2} \mathrm{O}_{2}$ & $3.5 \times 10^{9}$ & \\
\hline $\mathrm{R} 27$ & $\mathrm{O}_{2}^{-}+\mathrm{Cu}^{+}+\mathrm{H}^{+} \rightarrow \mathrm{Cu}^{2+}+\mathrm{H}_{2} \mathrm{O}_{2}$ & $9.4 \times 10^{9}$ & \\
\hline $\mathrm{R} 28$ & $\mathrm{HO}_{2}+\mathrm{Cu}^{2+} \rightarrow \mathrm{Cu}^{+}+\mathrm{O}_{2}+\mathrm{H}^{+}$ & $1.0 \times 10^{8}$ & \\
\hline $\mathrm{R} 29$ & $\mathrm{O}_{2}^{-}+\mathrm{Cu}^{2+} \rightarrow \mathrm{Cu}^{+}+\mathrm{O}_{2}$ & $8.0 \times 10^{9}$ & \\
\hline
\end{tabular}




\begin{tabular}{|c|c|c|c|}
\hline $\mathrm{R} 30$ & $\mathrm{HO}_{2}+\mathrm{CuSO}_{4} \rightarrow \mathrm{Cu}^{+}+\mathrm{O}_{2}+\mathrm{HSO}_{4}^{-}$ & $1.0 \times 10^{7}$ & \\
\hline $\mathrm{R} 31$ & $\mathrm{O}_{2}^{-}+\mathrm{CuSO}_{4} \rightarrow \mathrm{Cu}^{+}+\mathrm{O}_{2}+\mathrm{SO}_{4}^{2-}$ & $1.0 \times 10^{8}$ & \\
\hline $\mathrm{R} 32$ & $\mathrm{Fe}^{3+}+\mathrm{Cu}^{+} \rightarrow \mathrm{Cu}^{2+}+\mathrm{Fe}^{2+}$ & $1.3 \times 10^{7}$ & \\
\hline $\mathrm{R} 33$ & $\mathrm{Fe}(\mathrm{OH})^{2+}+\mathrm{Cu}^{+} \rightarrow \mathrm{Cu}^{2+}+\mathrm{Fe}^{2+}+\mathrm{OH}^{-}$ & $3.0 \times 10^{7}$ & \\
\hline R34 & $\mathrm{Fe}(\mathrm{OH})^{2+}+\mathrm{Cu}^{+} \rightarrow \mathrm{Cu}^{2+}+\mathrm{Fe}^{2+}+2 \mathrm{OH}^{-}$ & $1.3 \times 10^{7}$ & \\
\hline $\mathrm{R} 35$ & $\mathrm{Fe}\left(\mathrm{SO}_{4}\right)^{+}+\mathrm{Cu}^{+} \rightarrow \mathrm{Cu}^{2+}+\mathrm{Fe}^{2+}+\mathrm{SO}_{4}^{2-}$ & $1.8 \times 10^{6}$ & \\
\hline $\mathrm{R} 36$ & $\mathrm{OH}+\mathrm{HO}_{2} \rightarrow \mathrm{H}_{2} \mathrm{O}+\mathrm{O}_{2}$ & $7.0 \times 10^{9}$ & \\
\hline R37 & $\mathrm{OH}+\mathrm{O}_{2}^{-} \rightarrow \mathrm{OH}^{-}+\mathrm{O}_{2}$ & $1.0 \times 10^{10}$ & \\
\hline $\mathrm{R} 38$ & $\mathrm{OH}+\mathrm{H}_{2} \mathrm{O}_{2} \rightarrow \mathrm{H}_{2} \mathrm{O}+\mathrm{HO}_{2}$ & $2.7 \times 10^{7}$ & \\
\hline R39 & $\mathrm{HO}_{2}+\mathrm{HO}_{2} \rightarrow \mathrm{H}_{2} \mathrm{O}_{2}+\mathrm{O}_{2}$ & $8.6 \times 10^{5}$ & \\
\hline $\mathrm{R} 40$ & $\mathrm{HO}_{2}+\mathrm{O}_{2}^{-} \rightarrow \mathrm{H}_{2} \mathrm{O}_{2}+\mathrm{O}_{2}+\mathrm{OH}^{-}$ & $1.0 \times 10^{8}$ & \\
\hline $\mathrm{R} 41$ & $\mathrm{O}_{3}+\mathrm{O}_{2}^{-}+\mathrm{H}_{2} \mathrm{O} \rightarrow \mathrm{OH}+2 \mathrm{O}_{2}+\mathrm{OH}^{-}$ & $1.5 \times 10^{9}$ & \\
\hline $\mathrm{R} 42$ & $\mathrm{SO}_{2}+\mathrm{O}_{3}+\mathrm{H}_{2} \mathrm{O} \rightarrow \mathrm{HSO}_{4}^{-}+\mathrm{O}_{2}+\mathrm{H}^{+}$ & $2.4 \times 10^{4}$ & \\
\hline $\mathrm{R} 43$ & $\mathrm{HSO}_{3}^{-}+\mathrm{H}_{2} \mathrm{O}_{2}+\mathrm{H}^{+} \rightarrow \mathrm{SO}_{4}^{2-}+\mathrm{H}_{2} \mathrm{O}+2 \mathrm{H}^{+}$ & $6.9 \times 10^{7}$ & 4000 \\
\hline $\mathrm{R} 44$ & $\mathrm{HSO}_{3^{-}}+\mathrm{O}_{3} \rightarrow \mathrm{HSO}_{4}^{-}+\mathrm{O}_{2}$ & $3.7 \times 10^{5}$ & 5530 \\
\hline $\mathrm{R} 45$ & $\mathrm{SO}_{3}^{2-}+\mathrm{O}_{3} \rightarrow \mathrm{SO}_{4}^{2-}+\mathrm{O}_{2}$ & $1.5 \times 10^{9}$ & 5280 \\
\hline $\mathrm{P} 1$ & $\mathrm{O}_{3}+\mathrm{H}_{2} \mathrm{O} \rightarrow \mathrm{H}_{2} \mathrm{O}_{2}+\mathrm{O}_{2}$ & $2.98 \times 10^{-4}$ & \\
\hline $\mathrm{P} 2$ & $\mathrm{H}_{2} \mathrm{O}_{2} \rightarrow 2 \mathrm{OH}$ & $4.81 \times 10^{-6}$ & \\
\hline P3 & $\mathrm{Fe}^{3+}+\mathrm{H}_{2} \mathrm{O} \rightarrow \mathrm{Fe}^{2+}+\mathrm{OH}+\mathrm{H}^{+}$ & $6.41 \times 10^{-6}$ & \\
\hline $\mathrm{P} 4$ & $\mathrm{Fe}(\mathrm{OH})^{2+} \rightarrow \mathrm{Fe}^{2+}+\mathrm{OH}$ & $5.63 \times 10^{-3}$ & \\
\hline $\mathrm{E} 1$ & $\mathrm{Fe}^{2+}+\mathrm{H}_{2} \mathrm{O} \leftrightarrows \mathrm{Fe}(\mathrm{OH})^{+}+\mathrm{H}^{+}$ & $3.22 \times 10^{-10}$ & \\
\hline E2 & $\mathrm{Fe}^{3+}+\mathrm{H}_{2} \mathrm{O} \leftrightarrows \mathrm{Fe}(\mathrm{OH})^{2+}+\mathrm{H}^{+}$ & $6.0 \times 10^{-3}$ & \\
\hline E3 & $\mathrm{HO}_{2} \leftrightarrows \mathrm{O}_{2}^{-}+\mathrm{H}^{+}$ & $2.05 \times 10^{-5}$ & \\
\hline $\mathrm{E} 4$ & $\mathrm{H}_{2} \mathrm{O}_{2} \leftrightarrows \mathrm{HO}_{2}^{-}+\mathrm{H}^{+}$ & $1.6 \times 10^{-12}$ & -3700 \\
\hline E5 & $\mathrm{Cu}^{2+}+\mathrm{SO}_{4}^{2-} \leftrightarrows \mathrm{Cu}\left(\mathrm{SO}_{4}\right)$ & $2.3 \times 10^{2}$ & \\
\hline E6 & $\mathrm{HSO}_{4}^{-} \leftrightarrows \mathrm{SO}_{4}{ }^{2-}+\mathrm{H}^{+}$ & $1.02 \times 10^{-2}$ & -2700 \\
\hline E7 & $\mathrm{HSO}_{3}^{-} \leftrightarrows \mathrm{SO}_{3}{ }^{2-}+\mathrm{H}^{+}$ & $6.22 \times 10^{-8}$ & -1960 \\
\hline
\end{tabular}

${ }^{1} \mathrm{R}$ refers to reactions, $\mathrm{P}$ refers to photolysis, and $\mathrm{E}$ refers to equilibria. 
Table S2. Sensitivity of $\mathrm{HO}_{\mathrm{x}}$ and $\mathrm{H}_{2} \mathrm{O}_{2}$ concentration to aerosol optical depth (AOD).

\begin{tabular}{cccc}
\hline AOD & OH (pptv) & $\mathrm{HO}_{2}$ (pptv) & $\mathrm{H}_{2} \mathrm{O}_{2}$ (ppbv) \\
\hline 0 & 0.60 & 29.7 & 2.18 \\
1 & 0.26 & 17.0 & 0.38 \\
2 & 0.08 & 6.3 & 0.06 \\
3 & 0.03 & 4.5 & 0.03 \\
4 & 0.02 & 4.4 & 0.02 \\
5 & 0.01 & 4.8 & 0.03 \\
\hline
\end{tabular}




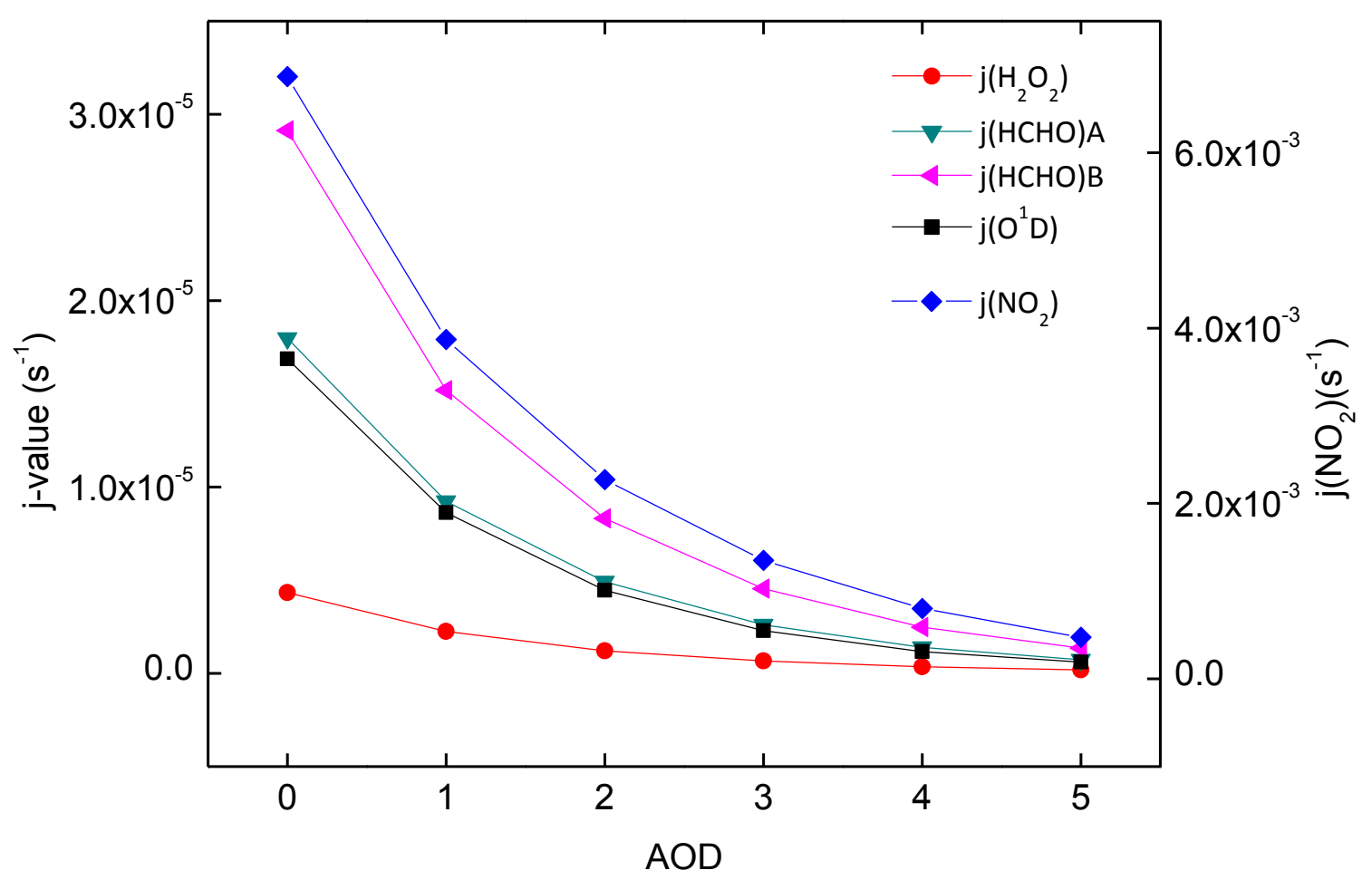

Fig. S1. Dependence of j-values on aerosol optical depth (AOD). 


\section{References}

Ervens, B., George, C., Williams, J. E., Buxton, G. V., Salmon, G. A., Bydder, M., Wilkinson, F., Dentener, F., Mirabel, P., Wolke, R., and Herrmann, H.: CAPRAM 2.4 (MODAC mechanism): An extended and condensed tropospheric aqueous phase mechanism and its application, J. Geophys. Res., 108, D14, doi: 10.1029/2002jd002202, 2003

Mao, J., Fan, S., Jacob, D. J., and Travis, K. R.: Radical loss in the atmosphere from $\mathrm{Cu}-\mathrm{Fe}$ redox coupling in aerosols, Atmos. Chem. Phys., 13, 509-519, doi: 10.5194/acp-13-509-2013, 2013. 